\title{
Molecular Dynamics of $\mu$ Opioid Receptor Complexes with Agonists and Antagonists
}

\author{
M. Kolinski and S. Filipek ${ }^{*}$
}

International Institute of Molecular and Cell Biology, Warsaw, Poland

\begin{abstract}
Opioid receptors like other G-protein-coupled receptors undergo specific rearrangements of structure upon activation by agonists. Such processes proceed via several steps ruled by different molecular switches. In order to reveal the first steps of receptor activation concurrent with ligand binding, we investigated $\mu$ opioid receptor complexes with antagonists - naltrexone and $\beta$-FNA, and two closely related agonists - morphine and N-methyl-morphine. The molecular dynamics simulations revealed distinct binding modes of analyzed antagonists and agonists. They all interacted with D3.32 on TM3 but while the antagonists formed a bond with Y3.33 (TM3) the agonists bound H6.52 (TM6). Furthermore, it was possible to observe a break of a hydrogen bond D3.32-Y7.43 between TM3 and TM7 (3-7 lock) during simulations of agonist complexes but not those of antagonist. Interdependence between the 3-7 lock and the rotamer toggle switch of W6.48 is proposed based on simulation of the naltrexone complex restrained to force an agonistic action.
\end{abstract}

\section{INTRODUCTION}

The large superfamily of $\mathrm{G}$ protein-coupled receptors (GPCRs) are essential for signaling across plasma membranes [1-3]. Each GPCR responds to a single or multiple ligands by activating $G$ proteins and thus giving rise to a highly amplified signaling cascade. GPCRs mediate responses to environmental stimuli such as light, taste and smell, but also to an enormous number of chemicals, hormones, peptides, small proteins and other ligands. For these reasons GPCRs are important targets for pharmacological intervention [4] and a large fraction of currently used drugs is directed toward them. GPCRs represent the largest and most versatile family of membrane receptors, and each member has a specific cellular life cycle and regulatory mechanisms [5]. Although so different in action all these receptors share the same topology - a bundle of seven transmembrane $\alpha$-helices - whereas the shapes and lengths of the $\mathrm{N}$ - and $\mathrm{C}$-termini as well as the cytoplasmic and extracellular loops are very different [1].

Opioid receptors belong to family A (rhodopsin-like) of GPCRs (this is a Rhodopsin group in the GRAFS classification system [6] based on phylogenetic studies - $\beta_{2} \mathrm{AR}$ also belongs to this group). They are located in the membrane of neurons of the central nervous system and of some types of smooth muscle cells. For the important role they play in the human body in controlling pain and stress, modulating immune responses and developing addiction the opioid receptors were subject of numerous investigations (see reviews [79]). There are four types of opioid receptors: $\mu, \delta, \kappa$ and also the nociceptin/opioid receptor like-1. They bind different sets of ligands but may share some of them. Drugs that interact with opioid receptors cause multiple effects including: analgesia, sedation, euphoria and physical dependence. Morphine, a common cause of opiate addiction, is a selective

*Address correspondence to this author at the International Institute of Molecular and Cell Biology, 4 Trojdena St, 02-109 Warsaw, Poland; Tel: (48-22) 5970-722; Fax: (48-22) 5970-715; E-mail: sfilipek@iimcb.gov.pl agonist of $\mu \mathrm{OR}$ and this receptor primarily mediates antinociceptive effects [10] but also participates in morphine mediated immune functions [11, 12]. Therefore, knowledge of precise mechanism of action of particular ligands will enable designing more potent ligands with fewer unwanted side effects.

There were many successful attempts of modeling ligand binding to various GPCRs to mention only the recent ones: Spijker et al. [13] using HierDock and Bhattacharya et al. [14] using a new method called LITiCon for $\beta_{2} \mathrm{AR}, \mathrm{Li}$ et al. [15] for the DP receptor, Heo et al. [16] for the MrgC11 receptor, Peng et al. [17] for the M1 muscarinic receptor, and Vaidehi et al. for the CCR1 chemokine receptor [18]. The theoretical methods may be used in an efficient way not only to obtain reliable models of complexes of GPCRs with their ligands but also for modeling activation processes (see reviews [19-21]). It was found that GPCRs exist in multiple conformational states that are in dynamic equilibrium. Transitions between these states are ruled by several conformational switches discovered first for the rhodopsin and $\beta_{2} \mathrm{AR}$, the best structurally described GPCRs [22-25]. The most important switches characterized to date involve E/DRY motif on TM3 linking TM3 and TM6, the CWxPxF sequence on TM6 forming the so called rotamer toggle switch, and the connection between TM3 and TM7 (not associated with specific sequence motif).

Activated receptor undergoes conformational rearrangements leading to transmitting the signal to cell interior. In order to get some insight into molecular mechanisms involved in these rearrangements the knowledge of the structures of GPCRs is required. The first GPCR structure determined was that of rhodopsin [26, 27], and recently, the structure of $\beta_{2}$ adrenergic receptor ( $\left.\beta_{2} \mathrm{AR}\right)$ was obtained [28-30]. The latter structure proved to be very similar to rhodopsin 3D structure in the transmembrane region. Also the ligand in the binding site was located nearly in the same position as retinal in rhodopsin. Rhodopsin was a template for homological building of multiple GPCRs and some of these models, including opioid receptors, were subjected to ligand 
docking and molecular dynamics of ligand-receptor complexes.

The homology modeling was used by Zhang et al. [31] to construct the $\mathrm{N}$ - and C-truncated $\mu$ opioid receptor ( $\mu \mathrm{OR}$ ) and $2 \mathrm{~ns}$ dynamics was conducted to optimize its structure. The ligand (naltrexone) was manually docked to the receptor binding pocket and 11 ps molecular dynamics (MD) simulation was applied to the complex. Homology modeling in combination with mutagenesis data was also used by Pogozheva et al. [32] to dock agonists and antagonists to all three types of opioid receptors but no simulations were conducted. In both studies the ligands were docked in the same way involving interactions with D3.32 on TM3 and H6.52 on TM6 for both agonists and antagonists (numbers according to the Ballesteros-Weinstein numbering scheme: every amino acid identifier starts with the helix number, followed by the position relative to a reference residue being the most conserved amino acid in that helix which bears number 50 [33]).

In this paper we modeled the structure of $\mu \mathrm{OR}$ to investigate changes in the receptor associated with binding with structurally similar and mostly rigid nonpeptide antagonists and agonists. All ligands were analogs of morphine i.e. built on a tyramine ( $p$-hydroxyphenethylamine) scaffold so the two parts, the "message" (tyramine) and the "address" [34], were well distinguished. Using simulated annealing procedure we found that the antagonists preferentially bound to Y3.33 whereas the agonists to H6.52. Moreover, during conducted molecular dynamics simulations we observed a break of a hydrogen bond D3.32-Y7.43 linking TM3 and TM7 in complexes with morphine and N-methyl-morphine and also in the complex with an antagonist, naltrexone, restrained to bind H6.52. An action of another switch, the rotamer toggle switch, was also observed and these both switches were nearly concurrent.

\section{METHODS}

Modeling the membrane. A model of the cell membrane composed of DPPC (dipalmitoyl-phosphatidylcholine) lipid bilayer was built in Gromacs (v. 3.3) [35]. The lipid bilayer was surrounded by water molecules in a periodic box $(6.4 \mathrm{~nm} \times 6.4 \mathrm{~nm} \times 9.5 \mathrm{~nm})$. The system contained 128 phospholipids (64 in each layer) and over 7000 water molecules. Optimization of the system was done at first by energy minimization, and then molecular dynamics (MD) was applied. A standard ffgmx forcefield with additional parameters for lipids [36] and water SPC [37] was used for more accurate treatment of hydrogen bonds. The PME procedure [38] was applied for treatment of the long-range electrostatic interactions. In the first step of MD lasting $500 \mathrm{ps}$, restraints were imposed on all phosphorus atoms in direction perpendicular to the membrane and the box dimensions were frozen to avoid breaking the premature membrane. In the second step, lasting $20 \mathrm{~ns}$, no restraints were applied and the membrane became equilibrated. MD was performed at the temperature of $310 \mathrm{~K}$, pressure of $1013 \mathrm{hPa}$, and the simulation time step was $1 \mathrm{fs}$.

Building of apoprotein. The $\mu$ opioid receptor structure modeling was done on the basis of the crystal structure of inactive rhodopsin [26] with no gaps (Protein Data Bank code 1U19) [27]. The Clustal W algorithm [39] was em- ployed for automatic alignment of multiple sequences. The final alignment is shown in Fig. (1) of the supporting material. The most conserved residues (numbered x.50) were aligned in all transmembrane helices. These are N1.50, D2.50, R3.50, W4.50 and proline residues of the remaining transmembrane helices. There are no gaps in the transmembrane helices (TMs) with the exception of TM2 where one glycine residue of rhodopsin was skipped in the corresponding opioid receptor sequence. This is a site with one additional amino acid in the turn of the rhodopsin $\alpha$-helix so the removal of one residue restored the regular structure of TM2 in $\mu \mathrm{OR}$. Relative to the $\beta_{2} \mathrm{AR}$ structure cysteine residues forming a disulfide bridge, C3.25 in TM3, were aligned properly whereas cysteines from the loop linking TM4 and TM5 were set in a different position using automatic alignment (see Fig. (1) in the supporting material). There is also a second pair of cysteine residues in $\beta_{2}$ AR forming a disulfide bridge and supporting a short helix instead of a $\beta$-sheet in rhodopsin. The microdomains important for activation of GPCRs, E/DRY, CWxP and NpxxY (including phenylalanine residue in the H8 helix supplementing the NPxxY motif by interacting with Y7.53 [40]), were properly aligned.

Homology modeling of the transmembrane part of $\mu \mathrm{OR}$ including all internal loops was done using Modeller [41, 42]. Differences in sequence and length of the N- and Ctermini make structural predictions of these regions difficult. The N- and C- termini protect the hydrophobic interior of the transmembrane domain bundle so it was necessary to model the whole-length receptor. For modeling of the long termini of $\mu$ OR we used the most similar proteins (found by SeqFold program based on sequence similarity). The $\alpha$-bungarotoxin structure (PDB code $2 \mathrm{ABX}$ ) was chosen by the program for modeling the N-terminus of $\mu \mathrm{OR}$ and the swi5 zinc finger protein structure (PDB code 1ZFD) for the C-terminus. Fitting both termini to the rest of the receptor was done on the basis of the best match of hydrophobic regions.

The palmitoyl chain was added to cysteine at the end of the cytoplasmic helix H8 (position 353). Then, the receptor model was inserted into the membrane. Excessive lipid molecules were removed. Counterions were added to make the system neutral (necessary for PME procedure). After energy minimization the three step simulations were performed. During the first step lasting $1 \mathrm{~ns}$ all backbone atoms were restrained to their initial positions, hydrogen bonds were also restrained with the LINCS algorithm [43] (other parameters remained the same as before for membrane simulation). In the second step (2 ns) restraints were removed from both termini and all loops of the receptor. In the third step (lasting also $2 \mathrm{~ns}$ ) all restraints were released.

Quality of opioid receptor models. For checking the quality of the obtained models the Procheck [44, 45] program was used. The Ramachandran plots obtained for the opioid receptor model and for the crystal structure of rhodopsin (used for comparison) are shown in Fig. (2) of the supporting material. To check the structure/sequence compatibility of the resulting receptor structures we used the Verify-3D method [46, 47]. For $\mu \mathrm{OR}$, the best selfcompatibility score for homology-modeled opioid receptor structures was 110. This value should be compared to the threshold value of the score for proteins of the same length, which was 82 . Therefore, the structure of $\mu \mathrm{OR}$ is character- 
ized by high compatibility score being well above the threshold. For comparison, the self-compatibility score for the crystal structure of rhodopsin (PDB code 1U19) is 120 with a threshold value of 73 .

Insertion of ligands and simulations. The placement of the $\mu$ opioid receptor ligands was done for two antagonists: NTX and $\beta$-FNA, and for two agonists - morphine and Nmethyl-morphine. The ligands were modeled in their protonated-nitrogen forms. Geometry optimization and calculations of the electrostatic potential was conducted using the Hartree-Fock procedure employing $6-31 \mathrm{G}^{*}$ basis set in Gaussian (v.03 rev. C.02 Gaussian Inc.). The atomic partial charges were obtained by fitting to electrostatic potential using RESP method [48]. The ligands were inserted in the middle of the cavity formed by helices to preserve the interaction between D3.32 and the protonated amine nitrogen atom present in all ligands. Phenolic $\mathrm{OH}$ groups of all analyzed agonists and antagonists were docked to form a hydrogen bond with H6.52 (similarly to the structures of complexes of opioid receptors constructed by Pogozheva et al. [32]).

In order to sample different possibilities of binding in the cavity of the receptor we subjected all created complexes to the cyclic simulated annealing procedure in Yasara (v.7.8, Yasara Biosciences) with Yamber2 forcefield [49] with temperature diminishing from $500 \mathrm{~K}$ to $0 \mathrm{~K}$ whereas the length of the cycle was variable. During this procedure the ligand and its $0.9 \mathrm{~nm}$ protein vicinity were allowed to move. Initial positions of ligands in a binding site were modified manually while preserving the salt bridge between charged amine group of a ligand and carboxyl group of D3.32. The optimized complexes were inserted into the DPPC membrane (taken from $5 \mathrm{~ns}$ simulations of empty opioid receptor) and subjected to MD simulations. In the first 100 ps simulation the restraints were imposed on the transmembrane (TM) part of the backbone of each receptor. In the second step of MD, lasting $2 \mathrm{~ns}$ for antagonists and $7.5 \mathrm{~ns}$ for agonists, all restraints were removed. Total time of all conducted MD simulations was about $80 \mathrm{~ns}$ since most of them (excluding equilibration of the membrane) were repeated several times.

The Modeller, Clustal W, SeqFold and Profile-3D (Verify-3D method) programs were used within the InsightII software platform (Accelrys Inc., v.2000.1). All figures showing molecular structures were drawn using MolMol [50] except Fig. (5) in the supporting material done in VMD [51]. Movies (Movies 1-4 in the supporting material) were created in VMD.

\section{RESULTS}

Molecular dynamics simulation of the apoprotein. To study changes in the receptor and the early transitions upon ligand binding in $\mu \mathrm{OR}$ we chose the crystal structure of inactive rhodopsin as a template for the homology modeling procedure. One additional residue in a helix turn in TM2, a feature present in the rhodopsin crystal structure, was removed because of the alignment whereas the one in TM5 was preserved. Such arrangements are in agreement with opioid receptor structures obtained by Fowler et al. [52] based on engineered zinc-binding sites.

\section{extracellular side}

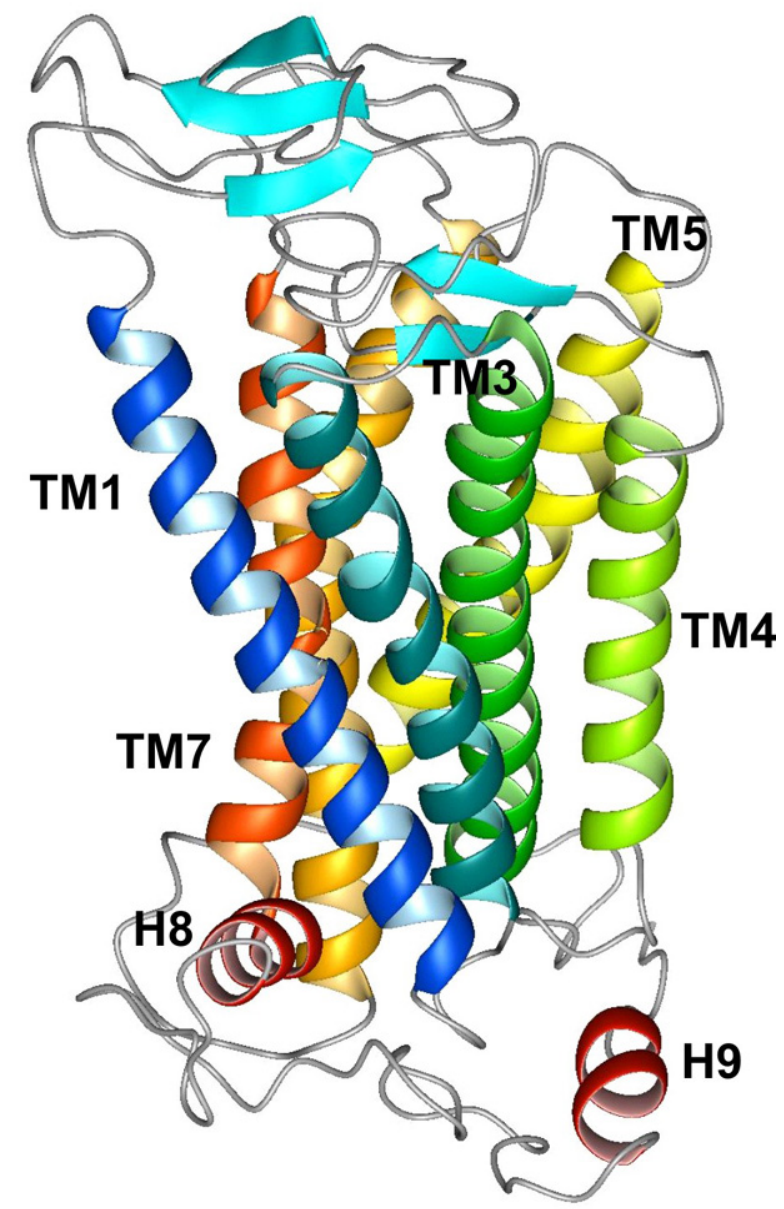

cytoplasmic side

Fig. (1). A model of the $\mu$ opioid receptor structure. Transmembrane helices are colored: TM1 blue, TM2 blue-green, TM3 green, TM4 yellow-green, TM5 yellow, TM6 orange, TM7 light-red. Cytoplasmic helices $\mathrm{H} 8$ and $\mathrm{H} 9$ are colored in red.

The structure of the apo $\mu$ opioid receptor after MD simulation in the membrane is shown in Fig. (1). It consists of a bundle of seven transmembrane helices and an additional amphiphilic helix H8 (present also in the crystal structure of $\left.\beta_{2} \mathrm{AR}\right)$. The extracellular loop connecting helices TM4 and TM5 is in a form of a $\beta$-sheet as it is in rhodopsin. The N-terminus of the $\mu \mathrm{OR}$ model contains an additional $\beta$ sheet, similarly to rhodopsin, although it was modeled based on a protein different from rhodopsin ( $\alpha$-bungarotoxin). This $\beta$-sheet is larger than in rhodopsin and is composed of three threads. The $\mathrm{C}$-terminus of $\mu \mathrm{OR}$ additionally contains a small 2-turn helix (H9). This part was modeled based on the swi5 zinc finger protein structure. Both "caps" of $\mu \mathrm{OR}$ were created for the completeness of the receptor structure but turned out to be good for protein structure quality and stable during MD simulations.

A second template that has become available recently is $\beta_{2} \mathrm{AR}$. There is an astonishing similarity in the location of transmembrane helices in the amino acid sequences (see Fig. 
(1) in supporting material) and in the 3D structures between this receptor and rhodopsin. This is, however, not the case for the rest of the structure. The $\mathrm{N}$ - and $\mathrm{C}$ - termini are not visible in the $\beta_{2} \mathrm{AR}$ crystal structure but the structure of a loop between transmembrane helices TM4 and TM5 is discernible and is significantly different in both receptors. There is a small $\alpha$-helix in $\beta_{2}$ AR instead of a $\beta$-sheet in rhodopsin. The existence of a second disulfide bridge is probably responsible for the preservation of this helix. Alignment shows (Fig. 2) that $\beta_{2} \mathrm{AR}$ is not a good template for modeling $\mu \mathrm{OR}$. Large rearrangements would be needed to model the TM4-TM5 loop based on $\beta_{2}$ AR. Short TM5TM6 loop in $\mu \mathrm{OR}$ and presence of only one disulfide bridge (two of them in $\beta_{2}$ AR stabilize the TM4-TM5 loop in helical conformation) makes the rhodopsin template more suitable for homology modeling of $\mu$ opioid receptor. The only distortion in the alignment of TM4-TM5 loop using rhodopsin template is a two amino acid shortening of the tip of a $\beta$ hairpin formed by the $\beta_{3}$ and $\beta_{4}$ threads. liganded receptor are shown in Fig. (3) of the supporting material. The values do not exceed $0.3 \mathrm{~nm}$ indicating that the cytoplasmic and extracellular "caps", composed of both termini and loops, were stable and compact during the whole simulation. The RMSD of TMs is nearly the same as for the intra- and extracellular caps and stabilizes at about $0.22 \mathrm{~nm}$. Both caps slightly change their structure (which is visible in their RMSD) at the end of the 2 ns simulation. The structure is very compact and stable in accordance with good quality results from both the Ramachandran analysis and the Verify3D method. The obtained distribution profiles of water, membrane and the receptor simulated in a periodic box are shown in Fig. (4) of the supporting material. The distribution profile of the membrane without protein, averaged for the 20 ns simulation, is shown in Fig. (4a) of the supporting material. All the plots show even distribution of the lipids in each leaflet of the bilayer. There is no water density in the hydrophobic part of the membrane indicating a stable and compact membrane system well suitable for membrane protein simu-
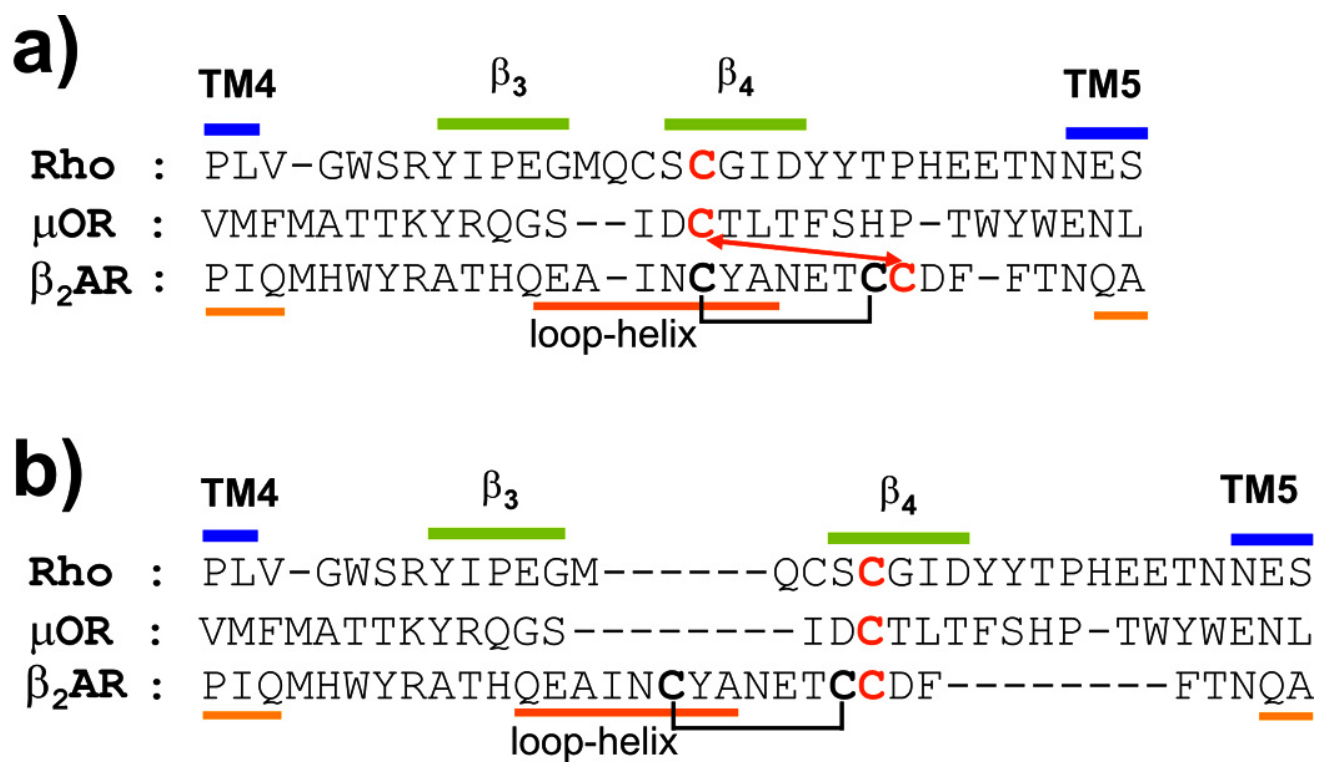

Fig. (2). Fragments of two alignments of rhodopsin, $\mu \mathrm{OR}$ and $\beta_{2} \mathrm{AR}$ containing a loop between TM4 and TM5. (a) automatic alignment. (b) corrected manual alignment. Additional disulfide bridge in $\beta_{2} A R$ is bracketed. Secondary structures in rhodopsin and $\beta_{2} A R$ are indicated. Bolded red cysteine residues are part of a disulfide bridge (bonded to TM3).

The sequential similarity (percent of identity) between $\mu \mathrm{OR}$ and rhodopsin is $19 \%$ for whole sequences and $24 \%$ for TMs, and between $\mu \mathrm{OR}$ and $\beta_{2} \mathrm{AR} 20 \%$ and $28 \%$, respectively. Both templates provide low similarity. Nevertheless, low sequential similarity does not lead to low structural similarity. The percent of identity between $\beta_{2} \mathrm{AR}$ and rhodopsin is only $22 \%$ for their TMs (18\% for whole sequences), but their structures are very similar: RMSD for TMs is only 0.21 $\mathrm{nm}$. We chose rhodopsin as a template because of better alignment of loops, however, after MD optimization, the structure of $\mu \mathrm{OR}$ differs from rhodopsin (RMSD $0.18 \mathrm{~nm}$ ) nearly the same amount as from $\beta_{2} \mathrm{AR}$ (RMSD $0.23 \mathrm{~nm}$ ).

Simulations of the $\mu$ opioid receptor model in the membrane. All features of the secondary structure were preserved during $\mathrm{MD}$ simulations of $\mu \mathrm{OR}$ in the membrane. Root mean square displacement (RMSD) plots for the last unrestrained $2 \mathrm{~ns}$ of MD simulation calculated for un- lations. The general view of the receptor within the membrane and of water in a periodic box is shown in Fig. (5) of the supporting material.

Binding of ligands to the receptor. In order to investigate similarities and differences in the binding of ligands we docked the nonselective antagonist naltrexone (NTX) and the selective one, $\beta$-funaltrexamine ( $\beta$-FNA), to $\mu \mathrm{OR}$. Both antagonists are analogs of morphine with an identical structural motif of tyramine (phenol and amine) (Chart 1) which is present in most of the nonpeptide opioid ligands. We also used morphine and N-methyl-morphine - $\mu \mathrm{OR}$ selective agonists. All ligands were manually placed in the cavity of the receptor close to the carboxyl group of D3.32 to set an interaction with the protonated nitrogen atom $\left(\mathrm{N}_{17}\right)$ in the tyramine structure. Phenolic $\mathrm{OH}$ groups of all analyzed agonists and antagonists were initially placed to form a hydrogen bond with H6.52. Such binding of ligands established 
their positions between three transmembrane helices TM3, TM6 and TM7 and also, partly, TM5. A general overview of the location of the ligands in the receptor is shown in Fig. (3). As it is explained in the Methods section, during the preliminary simulated annealing procedure the phenolic $\mathrm{OH}$ group $\left(\mathrm{C}_{3}\right)$ of agonists stayed bound to H6.52 whereas the same functional group in antagonists tended to bind to Y3.33, so we decided to explore further this effect.

\section{extracellular side}

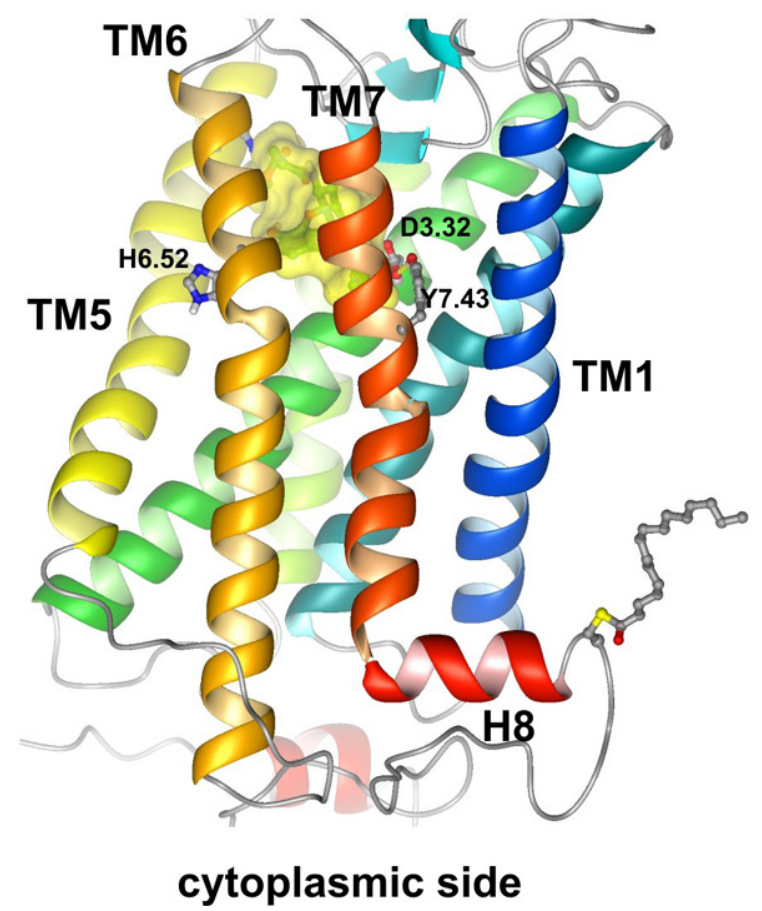

Fig. (3). General location of the ligands (here the biggest ligand, antagonist $\beta$-FNA, shown as a transparent yellow surface) in the binding pocket of $\mu \mathrm{OR}$.<smiles>COC(=O)/C=C/C(=O)NCCC12Oc3c(O)ccc4c3C1(CCN[C@@H]4CC1CC1)C2O</smiles>

$\beta$-FNA<smiles>C[NH+]1Cc2ccc(O)c3oc4c(O)ccc(c4c23)C1</smiles>

morphine<smiles>O=C1CC2(O)C3Cc4ccc(O)c5c4C2(CCN[C@@H]3C5)C1O</smiles>

NTX<smiles>CCN[C@@H]1CC(O)=C2CCc3c(O)ccc(c32)C1</smiles>

N-methylmorphine

Chart (1). Structural formulas of ligands used in this study.

Fig. (4). Details of the binding of $\mu \mathrm{OR}$ and (a) morphine (b) Nmethyl-morphine. View from the extracellular side. Carbon atoms of agonists are colored in orange and their phenolic rings are filled.
Binding of agonists. Both analyzed agonists, morphine and N-methyl-morphine, formed an ionic interaction between their protonated amine group and D3.32. In most of the stable conformations the second connection to the receptor was a hydrogen bond between a phenolic $\mathrm{OH}$ group of a ligand and a nitrogen atom of H6.52 (Fig. 4). However, such binding mode resulted in a break of the connection between transmembrane helices TM3 and TM7 and, more precisely, a break of a hydrogen bond between D3.32 and Y7.43. Such a bond (D3.32-Y7.43) exists and is stable in an empty opioid receptor. The plot of a distance D3.32-Y7.43 (Fig. 6 in the supporting material) reveals a stable hydrogen bond between these residues. There is a temporary (about $80 \mathrm{ps)} \mathrm{break} \mathrm{of}$ this hydrogen bond and a large increase in the distance between these residues. After inspection of the trajectory we

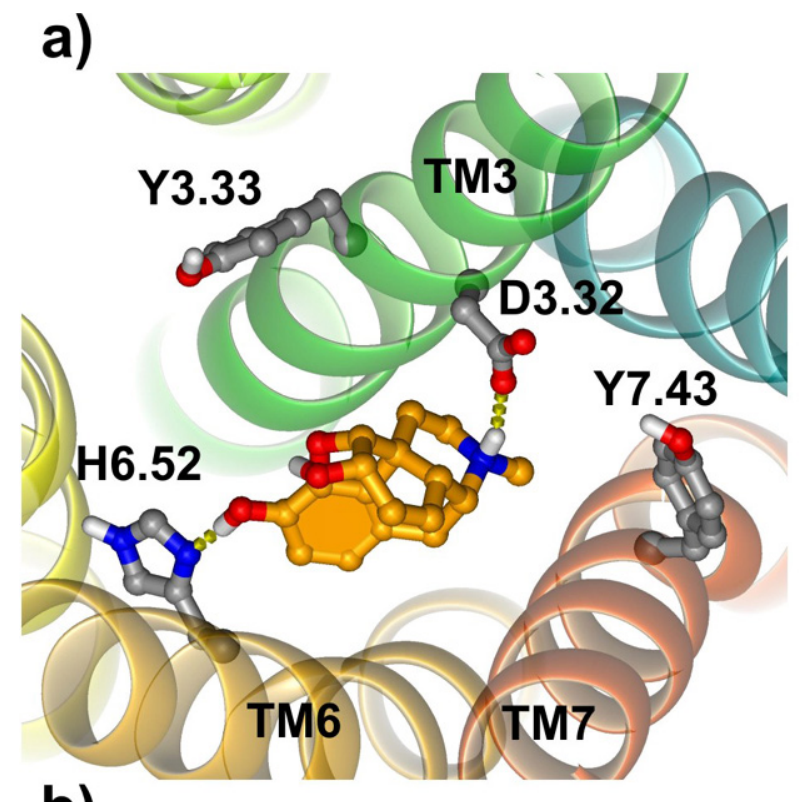

b)

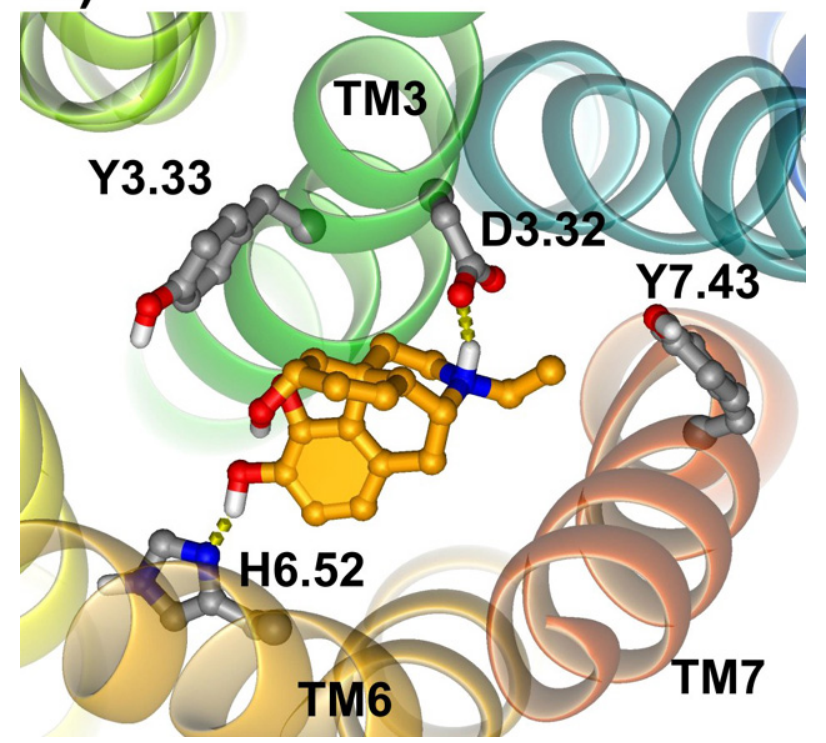


found that Y7.43 was moved away of its usual location towards TM1 and created a hydrogen bond with Y1.39. Y7.43 spontaneously came back to its position close to D3.32 and restored a hydrogen bond with it.

Other, much less frequent, conformations found in our study during simulated annealing procedure was the binding mode typical to antagonists i.e. binding of the tyramine hydroxyl group to Y3.33. To check preferences in the binding of agonists we performed a simulation with the phenolic $\mathrm{OH}$ group of morphine and N-methyl-morphine initially positioned in the middle of the distance between Y3.33 and H6.52. This group was initially interacting with both these amino acids. The ligands moved to a position close to H6.52 shortly after the simulations started (data not shown). For both agonists the connection D3.32-Y7.43 was broken during the simulations (Fig. 5) (Movies $\mathbf{1}$ and $\mathbf{2}$ in the supporting material). In the case of morphine it happened in a single fast event after about 1300 ps (Fig. 5a).

a)

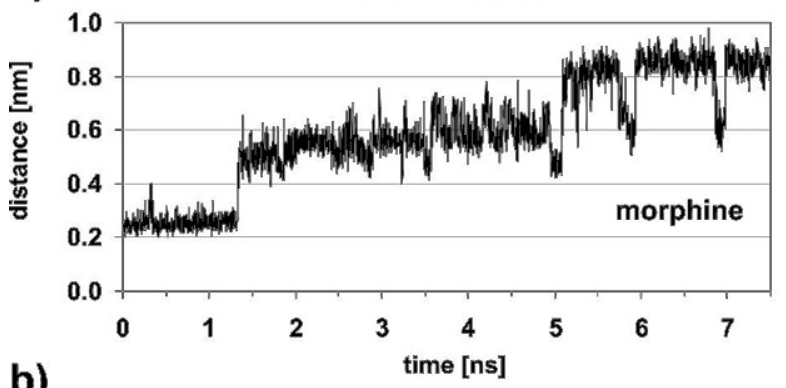

b)

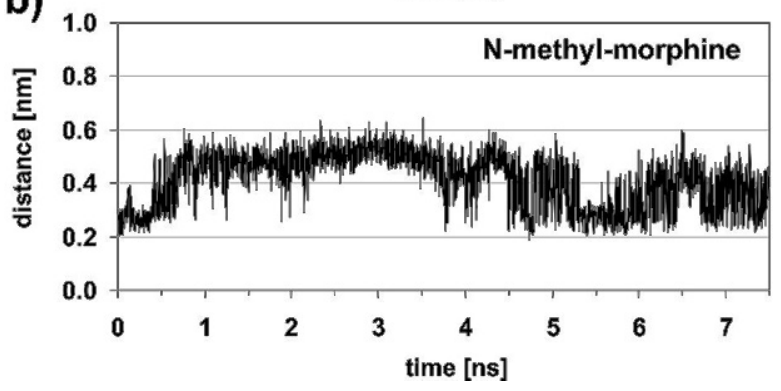

Fig. (5). D3.32-Y7.43 distance (between carboxyl and hydroxyl group) plots during MD simulation of $\mu \mathrm{OR}$ complexes with agonists. (a) Morphine $-\mu \mathrm{OR}$. (b) N-methyl-morphine $-\mu \mathrm{OR}$.

During later stages of the simulation the distance between D3.32 and Y7.43 increased even more. The hydrogen bond D3.32-Y7.43 was not restored even for a small period of time. The methyl group in N-methyl-morphine did not disturb location of this ligand in the binding site of the receptor but resulted in a much longer time required for breaking the D3.32-Y7.43 hydrogen bond (Fig. 5b). This process lasted from 400 ps to 700 ps of MD simulation and the average distance between D3.32 and Y7.43 was gradually increasing. The connection kept renewing for very short periods of time during the first $4.5 \mathrm{~ns}$. The process of restoration of this bond lasted from $4.5 \mathrm{~ns}$ to $5.2 \mathrm{~ns}$. The second breaking of this connection started at $5.7 \mathrm{~ns}$ and lasted to $6.5 \mathrm{~ns}$. Then, at $6.7 \mathrm{~ns}$ the next process of restoration started. In the case of morphine there were very fast changes of the distance D3.32-Y7.43 and longer periods of stability.

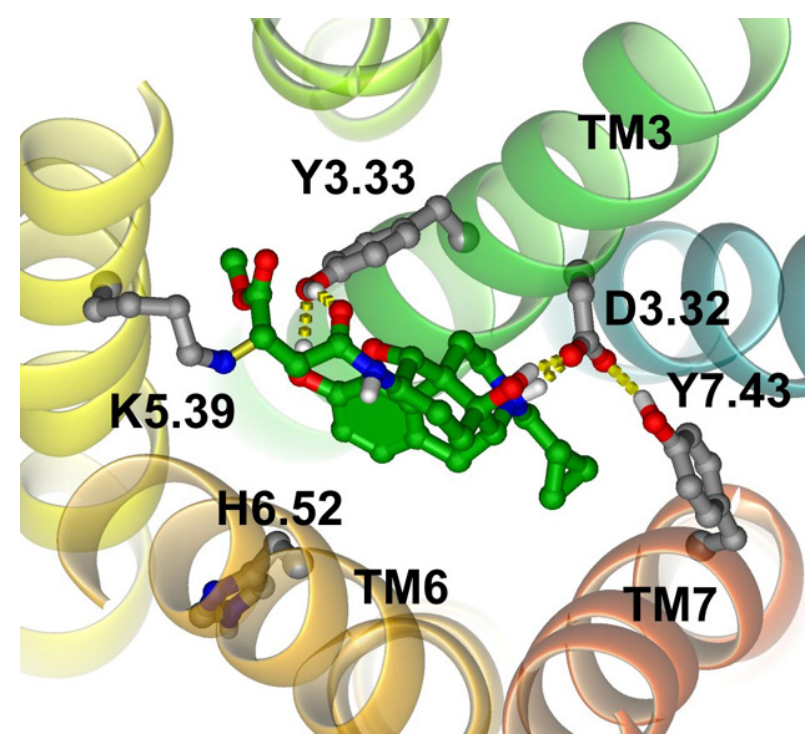

Fig. (6). The location and interactions of $\beta$-FNA in a binding pocket of $\mu \mathrm{OR}$. View from the extracellular side. Carbon atoms of the antagonist are colored in green and its phenolic ring is filled.
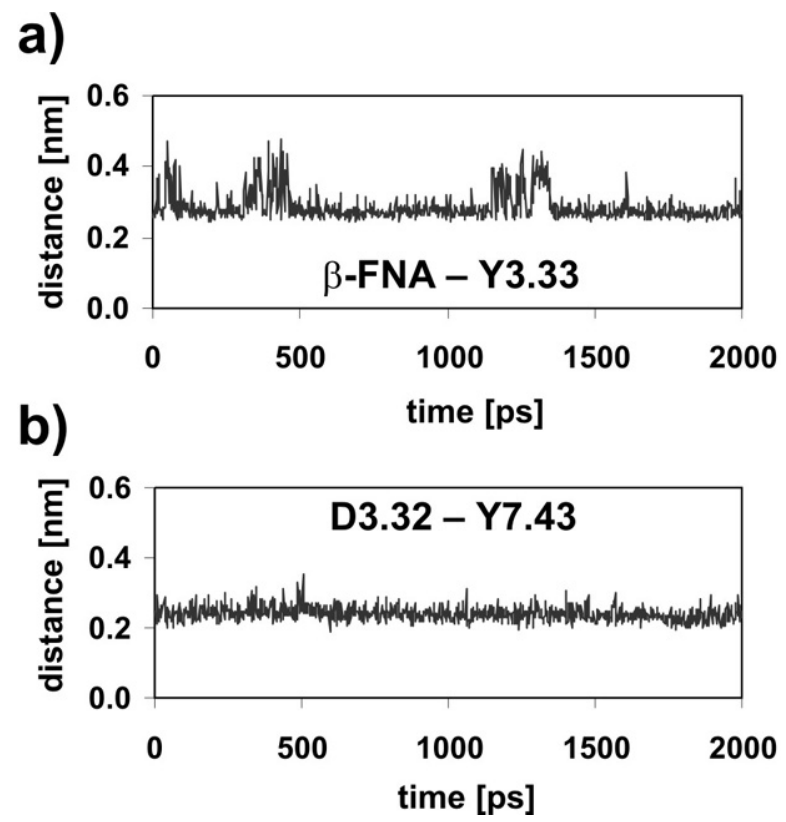

Fig. (7). Plots of distances (a) between the phenolic $\mathrm{OH}$ group of ligand and Y3.33, and (b) between D3.32 and Y7.43, from the simulation of the $\beta$-FNA $-\mu \mathrm{OR}$ complex.

Binding of antagonists. The biggest of the analyzed ligands was $\beta$-FNA. A stable position of $\beta$-FNA in the binding site was established between three transmembrane helices TM3, TM5, TM6 and TM7. A location of the ligand in the receptor and details of binding are shown in Fig. (6). $\beta$ FNA interacts with D3.32 and Y3.33 residues and these interactions remained unbroken during the whole simulation (see Fig. (7a) for a plot of a distance: ligand - Y3.33). We followed the experimental data on the formation of a covalent bond between $\beta$-FNA and K5.39. The covalently bound ligand formed an additional hydrogen bond between the carbonyl oxygen atom in its peptide bond and Y3.33. Both the 
protonated amine group and the $\mathrm{OH}$ group $\left(\mathrm{C}_{14}\right)$ were bound to the same oxygen atom of D3.32 whereas Y7.43 was bound to the second oxygen atom. The connection between transmembrane helices TM3 and TM7, a hydrogen bond D3.32-Y7.43, remained stable during simulation (Fig. 7b). This distance is undisturbed with a small amplitude, whereas the ligand - Y3.33 distance is disturbed by rare random spikes. It is because there is more room for the movement of Y3.33 than for Y7.43 in $\mu \mathrm{OR}$ and hence more possibilities to detach from the ligand.

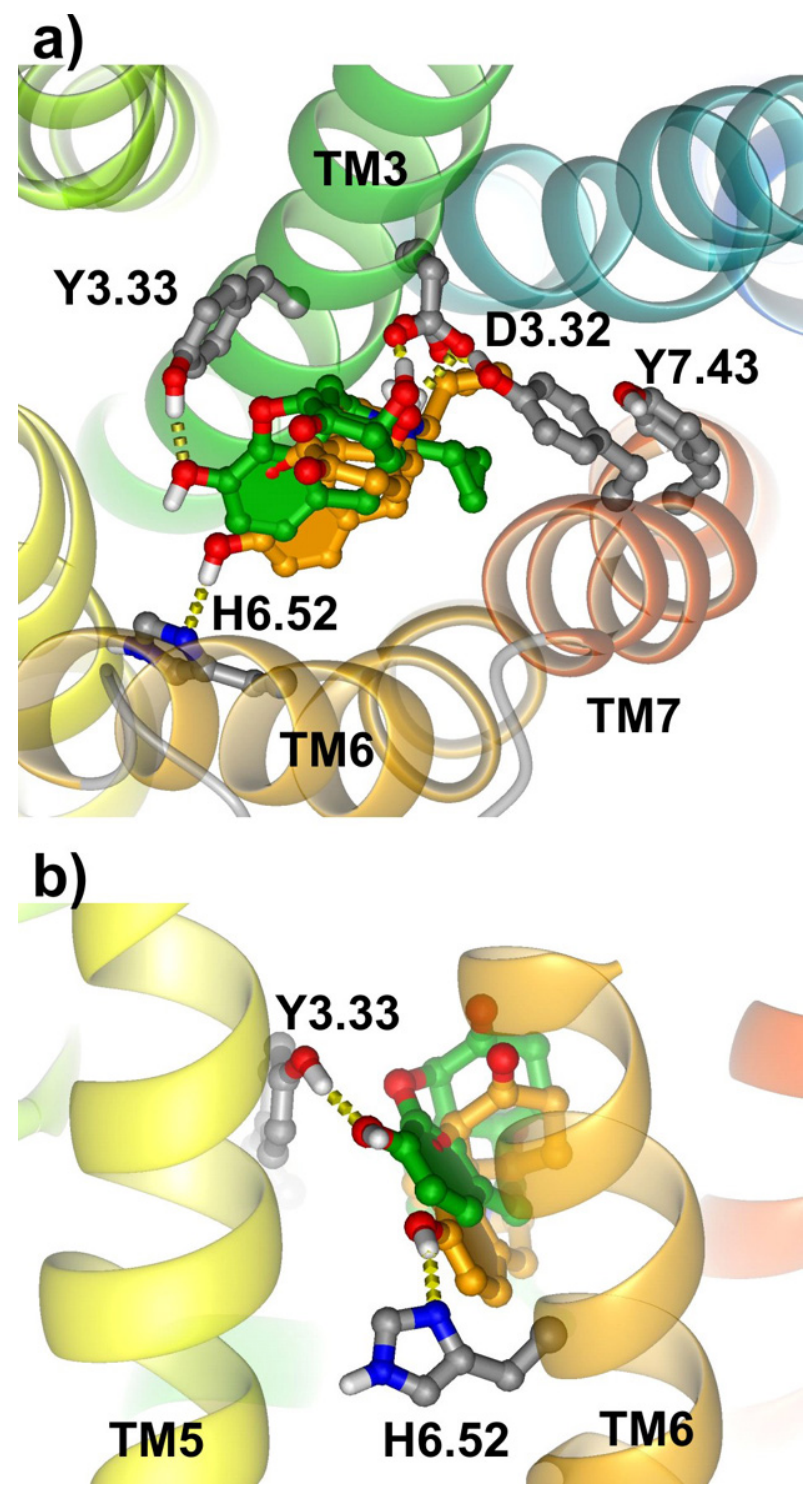

Fig. (8). The structures of NTX - $\mu \mathrm{OR}$ complexes from unrestrained (ligand in green) and restrained (ligand in orange) MD simulations. During the restrained simulation the connection between the phenolic $\mathrm{OH}$ group of the ligand and H6.52 was fixed to be $0.2 \mathrm{~nm}$. (a) view from the extracellular side. (b) a side view of the same structures.

The final position of the second analyzed antagonist, NTX, in the receptor was between three transmembrane helices TM3, TM6 and TM7 (NTX is colored in green in Fig. $(\mathbf{8 a}, \mathbf{b})$. This location was also characterized by two main interactions: that of the protonated amine nitrogen atom with D3.32 and of the phenolic OH group with Y3.33. During MD simulation the second $\mathrm{OH}$ group $\left(\mathrm{C}_{14}\right)$ of NTX formed a hydrogen bond with the carboxyl group of D3.32. The plots of the distance between the phenolic OH group of NTX and the $\mathrm{OH}$ group of Y3.33 and also of the distance D3.32-Y7.43 are shown in Fig. $(\mathbf{9 a}, \mathbf{b})$. All the plots indicate that the position of the ligand is stable (see also Movie $\mathbf{3}$ in the supporting material).

"Forced binding" of NTX. To check whether the transition of a ligand from binding Y3.33 to binding H6.52 is enough to break the hydrogen bond D3.32-Y7.43 we forced the nonselective antagonist NTX to behave like an agonist and to bind H6.52. In order to maintain such atypical location of NTX during simulation of the complex we imposed a weak harmonic restraint on the hydrogen bond between H6.52 and the phenolic OH group of NTX (the distance was $0.2 \mathrm{~nm}$ - the same distance was found in the morphine $\mu \mathrm{OR}$ complex). The initial position of NTX was taken from the final phase of the unrestrained simulation of this ligand what means that NTX was initially bound to Y3.33. The structure of the complex of NTX with a broken D3.32-Y7.43 connection can be seen in Fig. (8a,b) (ligand in orange).

During the simulation of NTX forced to bind H6.52 the hydrogen bond D3.32-Y7.43 broke (Fig. 10a) (see also Movie 4 in the supporting material). Breaking of this bond occurred only after a certain time although the hydrogen bond between NTX and H6.52 was created shortly after the beginning of simulation (data not shown). The time required to break D3.32-Y7.43 was about 400 ps. This break pushed NTX to change its structure as can be seen in Fig. (10b) showing a dihedral angle in NTX (rotation around a bond involving $\mathrm{N}_{17}$ and the first carbon atom from the hydrophobic tail holding the methyl-cyclopropane ring). This angle changed from $175^{\circ}$ to $155^{\circ}$ on average. At about 1200 ps this hydrophobic tail started to change its conformation, and the angle changed to $75^{\circ}$. However, it did not influence a rotamer of W6.48. Finally, at about 1750 ps the pressure of NTX on W6.48 resulted in a rotamer change of the tryptophan residue (Movie 4 in the supporting material). The $\chi_{2}$ angle of W6.48 changed from about $-65^{\circ}$ (vertical position) to $+85^{\circ}$ (horizontal position - perpendicular to the axis of TM6) (Fig. 10c). This rotamer swap is manifested not only by a change of the $\chi_{2}$ angle but also of the $\chi_{1}$ angle of W6.48 (from $65^{\circ}$ to $135^{\circ}$ ) and of the out-of-plane dihedral angle between the $\mathrm{C}_{\beta}-\mathrm{C}_{\gamma}$ bond and the plane of the pyrrole ring $\left(0^{\circ}\right.$ $\rightarrow-15^{\circ} \rightarrow 25^{\circ}$ ) (Fig. 7a,b in the supporting material, respectively). There is also a break of the hydrogen bond W6.48 S7.46 (Fig. (7c) in the supporting material) associated with W6.48 rotamer change.

\section{DISCUSSION}

Binding modes of ligands within $\mu \mathrm{OR}$. To reveal binding modes of ligands based on a tyramine structure within the $\mu$ opioid receptor we analyzed ligand-receptor complexes of two agonists, morphine and N-methyl-morphine, of a selective antagonist $\beta$-FNA and a nonselective (not having the "address" part) antagonist NTX. All of them were subjected to simulated annealing and molecular dynamics simulations. Simulation techniques helped us to investigate not only the 
a)
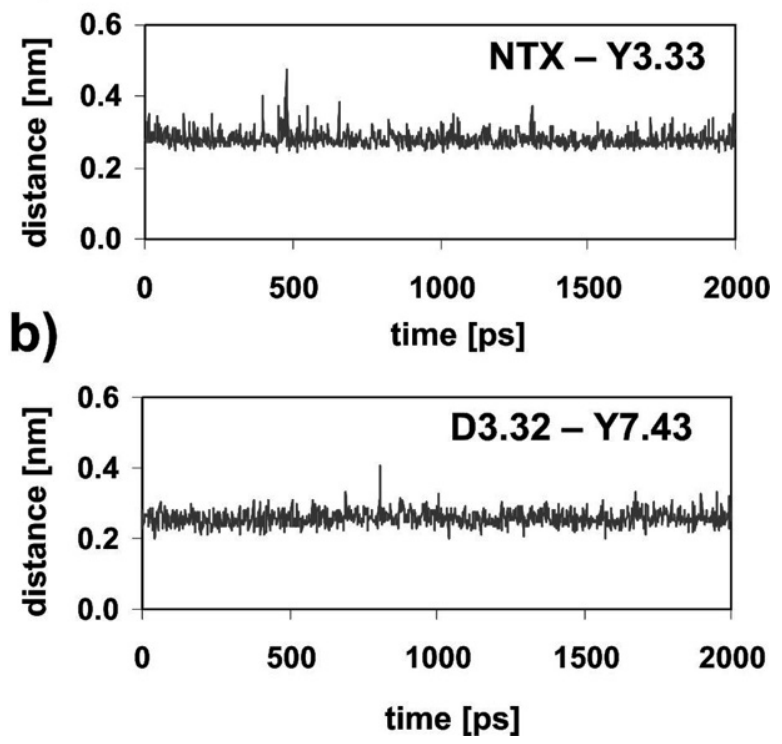

Fig. (9). Plots of distances (a) between the phenolic OH group of the ligand and Y3.33, and (b) between D3.32 and Y7.43, obtained from the simulation of the NTX $-\mu \mathrm{OR}$ complex.

ligand binding modes but also reorganization of the receptor structure upon ligand binding. Since we focused on the dynamical aspects of binding of agonists and antagonists we were interested also in outlining the very first steps of receptor activation. This is why we have chosen the inactive structure of rhodopsin as a template for building the $\mu$ opioid receptor structure. The whole process of receptor reorganization during activation is very complicated and still mostly unknown. Here we concentrated on these events which are concurrent with early steps of ligand binding in the binding pocket of the receptor.

For all analyzed ligands the protonated nitrogen atom $\left(\mathrm{N}_{17}\right)$ in the tyramine structure was bound to the carboxyl group of D3.32. Such binding mode is in agreement with many experimental studies and also with modeling research such as that of Zhang et al. (the Portoghese group) [31] and Pogozheva et al. (the Mosberg group) [32]. We found that the second binding anchor was different for the analyzed antagonists and agonists. Antagonists tended to bind to Y3.33 whereas agonists to $\mathrm{H} 6.52$ with their phenolic $\left(\mathrm{C}_{3}\right) \mathrm{OH}$ group. The distance between the phenolic $\mathrm{OH}$ group of antagonists and H6.52 is big enough to prevent creating a hydrogen bond. Additionally, the directionality of $\mathrm{C}_{\alpha}$ to $\mathrm{C}_{\beta}$ bonds in Y3.33 and H6.52 points the former residue up toward the extracellular side of a receptor whereas the latter one points down toward the cytoplasmic side. As can be seen from Fig. $(\mathbf{8 a}, \mathbf{b})$ the movement from Y3.33 (position found for antagonists) to H6.52 (position found for agonists) is not only horizontal (from TM3 to TM6) but also vertical and agonists penetrate deeper into the receptor structure. This deeper position of agonists (or "forced" antagonist) can induce a series of transitions in the receptor. A scheme illustrating proposed binding modes of antagonists and agonists as well as suggested first steps of agonist action is shown in Fig. (11). a)
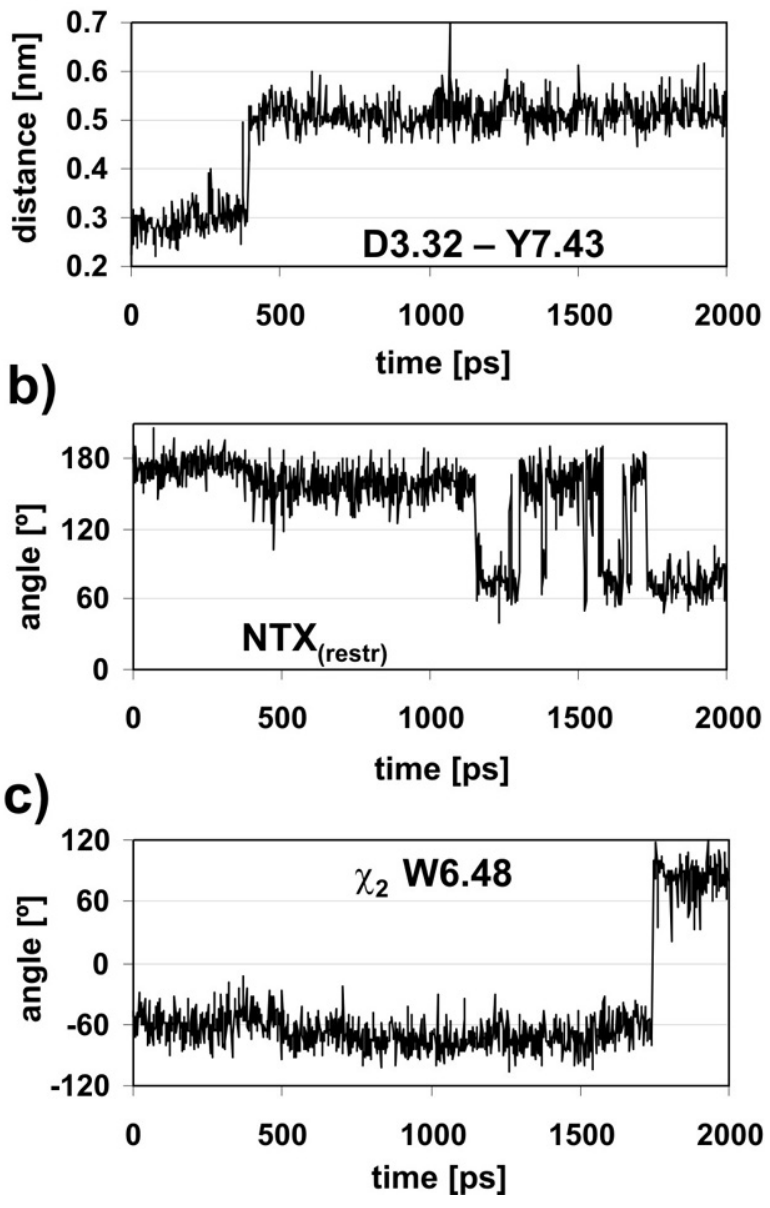

Fig. (10). Plots from the simulation of the NTX - $\mu$ OR complex with a restrained connection between the phenolic $\mathrm{OH}$ group of NTX and H6.52. (a) a distance between D3.32 and Y7.43. (b) a dihedral angle in NTX (rotation around bond $\mathrm{N}_{17}-\mathrm{C}_{\text {tail }}$ ). (c) the $\chi_{2}$ angle (rotation around bond $\mathrm{C}_{\beta}-\mathrm{C}_{\gamma}$ ) of W6.48.

Dynamics of agonist binding. Based on differences between the behavior of agonists and antagonists found in our simulations one can suggest the most probable way of ligand binding and propose the first steps of receptor activation. Both agonists and antagonists bind to Y3.33 but only agonists are able to rotate and reach $\mathrm{H} 6.52$ which is located deeper within the ligand pocket. The existence of such transition is consistent with experimental results [53] showing that mutations $\mathrm{Y} 3.33 \mathrm{~F}$ and $\mathrm{Y} 3.33 \mathrm{~A}$ reduce binding affinities of both agonists and antagonists. It means that also agonists can transiently bind to Y3.33. During the movement of the agonist both Y3.33 and H6.52 may form hydrogen bonds with its phenolic $\mathrm{OH}$ group. As a result of the agonist movement from Y3.33 to H6.52 the hydrogen bond D3.32Y7.43 gets broken. The agonist rotates around D3.32 (position of this residue remains the same - see Fig. (8a) of unrestrained and restrained NTX- $\mu \mathrm{OR}$ complexes) in the binding site and at the same time goes deeper into the receptor structure (Fig. 8b). Only NTX was used to study the forced binding to the opioid receptor since it has no "address" part, as is the case for selective antagonists, so its relocation is not 
connected to any other rearrangement in the receptor structure. Furthermore, NTX did not interact with the TM4-TM5 loop so eventual incorrect conformation of this loop in the modeled $\mu \mathrm{OR}$ did not affect conducted simulations.

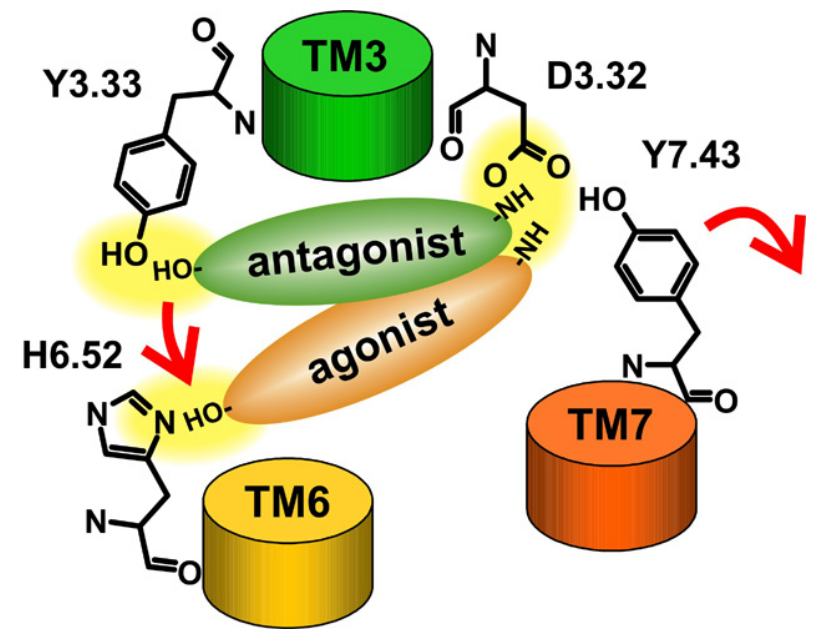

Fig. (11). A scheme of proposed binding modes of antagonists and agonists in opioid receptors. Red arrows indicate the suggested steps of agonist action. The agonist changes its location from Y3.33 to H6.52 going deeper into the receptor active site. As a result of this movement the hydrogen bond between D3.32 and Y7.43 is breaking.

Spivak et al. [54] found that mutation of the key residue H6.52 to glutamine or asparagine converts antagonists and inverse agonists (with structure based on the tyramine scaffold) into agonists of $\mu \mathrm{OR}$. According to our modeling study the flexible side chains of glutamine or asparagine may weaken the hydrogen bond between the phenolic $\mathrm{OH}$ of the ligands and Y3.33 and induce a movement toward position 6.52. This finally leads to the break of the D3.32-Y7.43 bond and possibly allows for subsequent activation steps.

Mechanism of GPCR activation. Role of switches. The mechanism of full activation of a GPCR is a complex process and possibly includes many switches [23, 24, 55]. The switches recognized so far are the DRY lock (break of a connection between TM3 and TM6) and the CWxP rotamer toggle switch. Additional potential switches may involve the NPxxY lock (break of a connection between TM7 and H8) [40] and recently discovered group-conserved residues [56]. These switches are not necessarily interdependent and different agonists and partial agonists may stabilize, fully or partially, the active state. In unliganded receptor the inactive state is stabilized by nonbonded interactions between transmembrane helices but also by specific conformations of side chains in the rotamer toggle switch. Distinct ligands are able to break or modify these stabilizing interactions leading to differential activation of the receptor.

The connection between TM3 and TM6 in $\mu \mathrm{OR}$ is established by R3.50 (the most conserved residue in the helix) and T6.34. There is no glutamic acid residue in TM6 to form a salt bridge with R3.50 as is the case in rhodopsin and $\beta_{2} \mathrm{AR}$ so another residue substitutes for it. The same residues, R3.50 and T6.34, participated in this connection in the Mosberg models of $\mu \mathrm{OR}$ complexes with antagonists [32]. In our simulations the TM3-TM6 link stayed unbroken (Fig. 12) for both agonists and antagonists. Stability of the TM3-TM6 connection confirms that our investigations were confined to the initial steps of receptor activation simultaneous with ligand binding. One of the events associated directly with binding of agonists proved to be a change of a state of the rotamer toggle switch. Both agonists changed the rotamer of W6.48 to a horizontal position (perpendicular to TM6) already in the simulated annealing phase so the subsequent MD simulations started with this switch in the ON state (Fig. 13). All antagonists maintained the initial vertical position of W6.48 in simulated annealing as well as in MD simulations. However, it was possible to observe an action of the rotamer toggle switch when forcing NTX to bind to the receptor binding pocket utilizing the agonist binding mode (a change of binding from Y3.33 to H6.52).

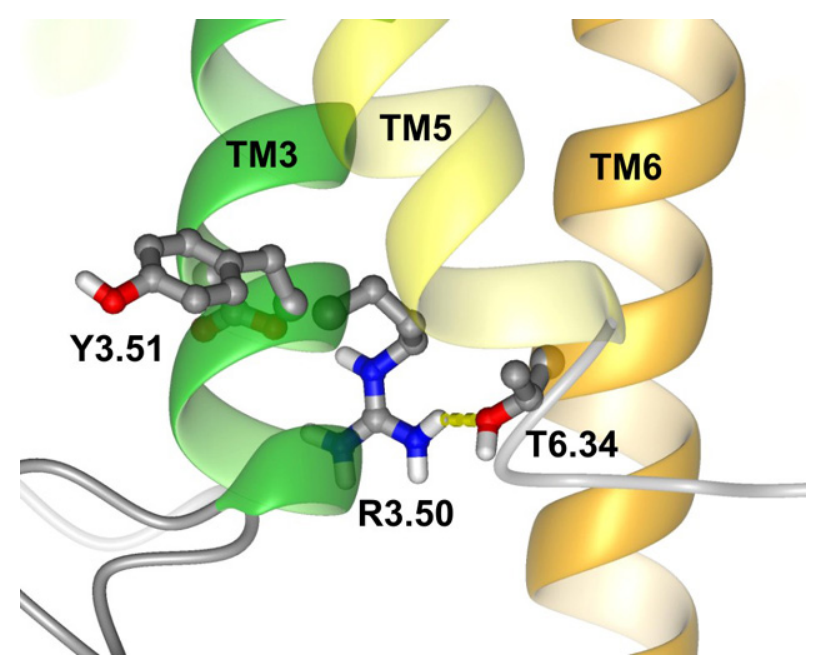

Fig. (12). Interactions in the switch area located at cytoplasmic ends of TM3 and TM6 in $\mu$ OR. Unbroken link between these helices indicates inactive state of this switch. The switch involves DRY motif from TM3.

Conformational response of the rotamer toggle switch involves changes in the position of aromatic amino acids surrounding the highly conserved proline P6.50 in TM6. This rotameric change, known as the rotamer toggle switch, has been proposed to be involved in the activation of amine and opsin receptor families $[57,58]$. It was proposed that rotamer configurations of $\mathrm{C} 6.47, \mathrm{~W} 6.48$ and $\mathrm{F} 6.52$, the residues that comprise the rotamer toggle switch in $\beta_{2} \mathrm{AR}$, are coupled and modulate the bend angle of TM6 around the kink at P6.50.

An additional switch involving breaking the connection between TM3 and TM7 was proposed by the Khorana group [55] based on mutagenesis experiments on rhodopsin. According to this research a salt bridge E113-K296 (E3.28K7.43) linking helices TM3 and TM7 in rhodopsin is a key constraint maintaining the resting state of the receptor. The paper also provides structural evidence that the disruption of the salt bridge is the cause rather than a consequence of the TM6 motion which occurs upon activation. The authors demonstrated that the K296-E113 connection may be a switch that, when broken through mutagenesis, results in a movement of TM6 similar but not identical to that caused by photoactivation of the WT receptor. Their data also indicate 
that the salt bridge is a primary constraint that determines the position of the cytoplasmic region of TM6. However, by itself, the salt bridge opening is insufficient to activate the receptor, and additional structural changes of a ligand and receptor are required for full activation. Such 3-7 lock (in the form of D3.32-Y7.43 hydrogen bond in opioid receptors) was postulated also by Befort et al. [59] to be one of the stabilizing factors of an inactive state of opioid receptors (mutation of D3.32 or Y7.43 in the delta opioid receptor resulted in constitutive activation of the receptor).

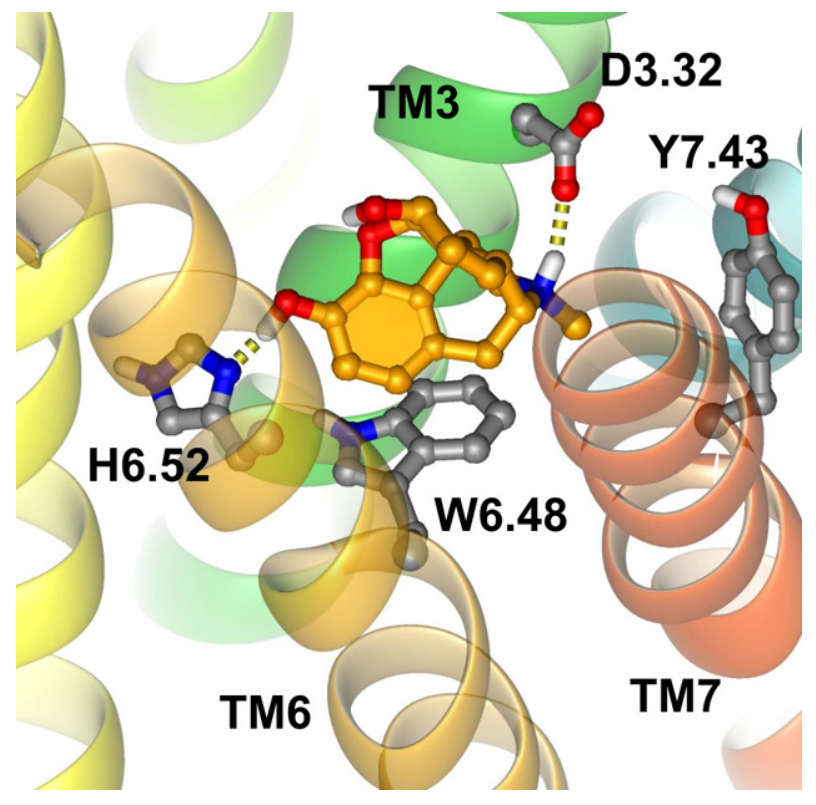

Fig. (13). Horizontal position of W6.48 interacting with morphine. The ligand is bound to H6.52 and 3-7 lock is broken.

Vilardaga et al. [60] suggested, based on fluorescence experiments on the $\alpha_{2 \mathrm{~A}}$-adrenergic receptor, that agonists and inverse agonists use distinct molecular switches in the receptor. However, in a recent crystal structure of $\beta_{2} \mathrm{AR}$ [29] the DRY lock (TM3-TM6) is broken upon binding of an inverse agonist - carazolol. It means that inverse agonists may use the same switches as agonists in combination with their specific switches that are still unknown. Yao et al. [61] investigated differences in the binding of full and partial agonists to $\beta_{2} \mathrm{AR}$. They found that both kinds of ligands disrupted the ionic bridge between the cytoplasmic ends of TM3 and TM6 but this was not enough for the full activation of the receptor by partial agonists. It was also shown for the $\beta_{2}$-adrenergic and $V_{2}$ vasopressin receptors that inverse agonists can also recruit $\beta$-arrestin (similarly to agonists) [62]. Moreover, inverse agonists of $\delta \mathrm{OR}$ in the cyclase pathway induced agonist responses in the ERK cascade [63]. It means that inverse agonist binding does not lead to full inactivation of a receptor but rather to alternative activation states. These data point to a multistate model of receptor activation in which ligand-specific conformations are capable of differentially activating distinct signaling partners.

Sequence of events. The action of two switches was observed in our simulations: break of the 3-7 lock and rotamer change of W6.48. The TM3-TM7 connection is formed in $\mu \mathrm{OR}$ by D3.32 and Y7.43. This aspartic acid residue exists also in $\beta_{2} \mathrm{AR}$ where is a key residue binding the charged amine nitrogen atom from a ligand. However, in rhodopsin, instead of D3.32, there is E3.28 which is located one turn of helix farther from K7.43 (which covalently binds retinal). $\mathrm{K} 7.43$ is in the same position in rhodopsin as Y7.43 in $\mu \mathrm{OR}$ or $\beta_{2} \mathrm{AR}$ (see alignment in Fig. (1) in the supporting material) but due to a longer side chain of glutamic acid a salt bridge between them is still possible. This glutamic acid residue is a counterion for the charged Schiff base of retinal so it has the same role as D3.32 for ligand binding in $\beta_{2} A R$ and in opioid receptors. During retinal isomerization a break of this salt bridge occurs because of a rotation of a part of retinal containing the Schiff base. The TM3-TM7 connection in the form D3.32-Y7.43 also exists in $\beta_{2} \mathrm{AR}$ and is clearly seen in a recent crystal structure of this receptor.

During conducted simulations both analyzed agonists broke the D3.32-Y7.43 hydrogen bond. However, none of the analyzed antagonists was able to break this hydrogen bond in MD simulations. An additional MD simulation was performed for the $\mu \mathrm{OR}$ - NTX complex with the NTX H6.52 hydrogen bond restrained to force an agonist-like binding mode. During this simulation we observed a characteristic sequence of events (Fig. 10a-c) linking disruption of the 3-7 lock with the rotamer toggle switch.

A restrained bond of NTX $\left(\mathrm{C}_{3}\right) \mathrm{OH}-\mathrm{H} 6.52$ was created shortly after MD simulation started although the initial position of NTX was close to Y3.33 (Fig. 14a). NTX rotated in the binding site to reach $\mathrm{H} 6.52$ but this rotation did not result in introducing any strain to its conformation as can be judged by an unchanged conformation of its flexible tail (Fig. 10b). No strain was also imposed on W6.48: its dihedral angles remained unchanged (Fig. (10c) and Fig. (7a) in the supporting material) and an out-of-plane dihedral angle between the $\mathrm{C}_{\beta}-\mathrm{C}_{\gamma}$ bond and a plane of the pyrrole ring was still zero (Fig. (7b) in the supporting material). A new position of the ligand resulted in a break of the 3-7 lock at about $400 \mathrm{ps}$. Only this event introduced strain on W6.48 (out-of-plane angle changed to $-15^{\circ}$ ) and also the conformation of the NTX slightly changed (Fig. 10b). NTX tended to move toward the empty space created between TM3 and TM7 but met an obstacle in the form of a bulky side chain of the tryptophan residue W6.48. This close encounter changed conformations of both NTX and W6.48. The flexible chain of NTX tended to change its conformation several times which indeed happened at about 1750 ps when a rotamer of W6.48 swapped from a vertical to horizontal position (perpendicular to axis of TM6) (Fig. 14b). During this swap the strain was not released from tryptophan residue but it even increased (out-ofplane angle changed to $+25^{\circ}$ ) (Fig. (7b) in the supporting material). A similar out-of-plane angle of W6.48 was found in the structures of $\mu \mathrm{OR}$ complexes with morphine (Fig. 13) and N-methyl-morphine where this W6.48 rotamer was created already during simulated annealing phase because of the inclination of these ligands to bind to H6.52. Such strain in W6.48 conformation is probably the reason for a change of position of the whole TM6 either by rotation or a seesaw motion.

The difference of about $1 \mathrm{~ns}$ between a break of the TM3-TM7 connection and the rotamer swap in W6.48 is very small compared to millisecond timescale of protein rearrangements. Therefore it is plausible to regard these two transitions as part of a single, larger switch. In our simulation 
a break of the 3-7 lock preceded the change of the rotamer but it may not be the same with other ligands so subsequent simulations are required to establish the correct timeline of these transitions. Certainly, in the case of $\mu \mathrm{OR}$, the first event is imposed by an agonist and we propose that this is a change of a binding mode of a ligand from Y3.33 to H6.52. It is followed by subsequent changes of the receptor structure: a break of a 3-7 lock and a rotamer swap of W6.48. It is to determine whether these two switches are interdependent also for other ligands.

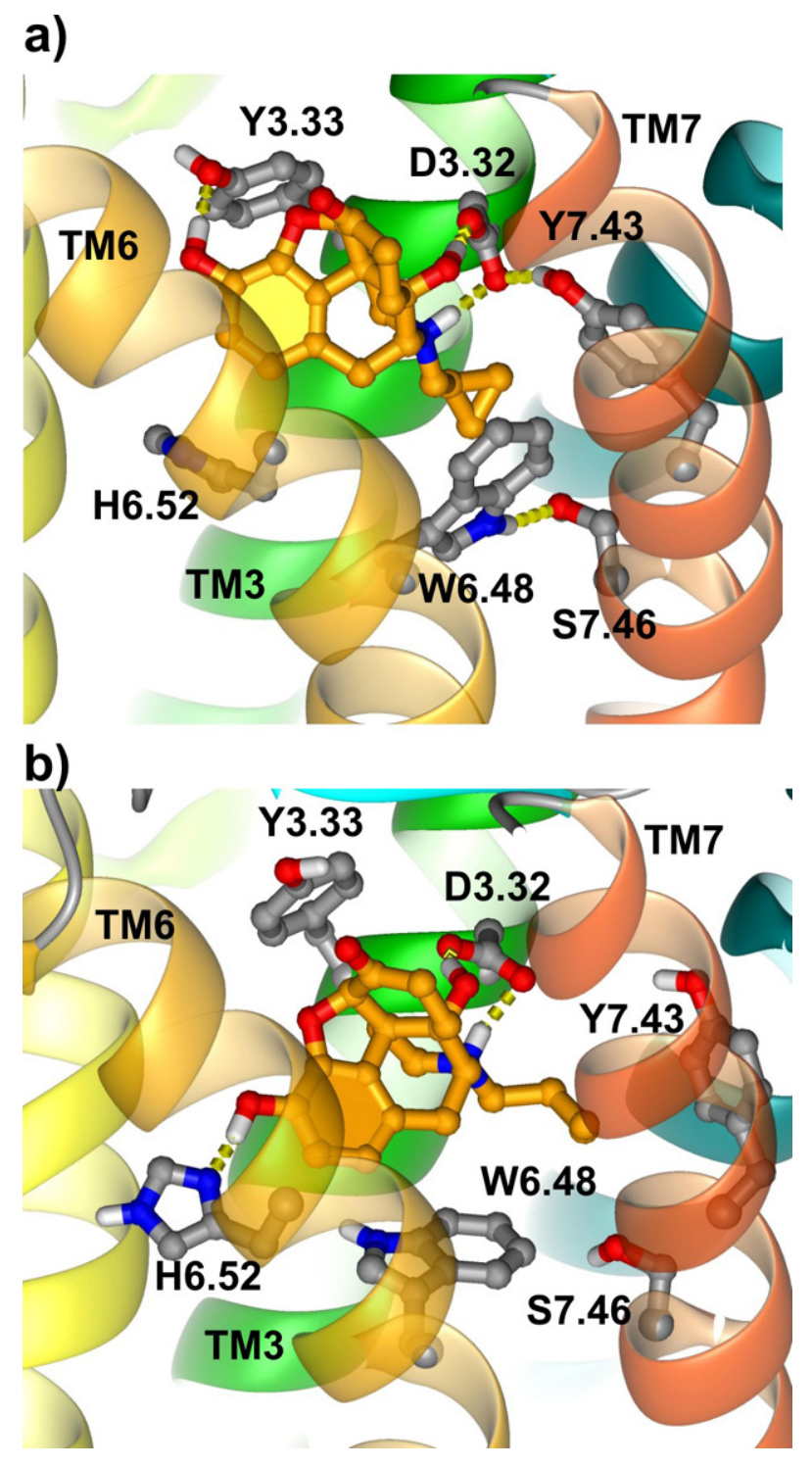

Fig. (14). Conformations of W6.48 in restrained NTX- $\mu$ OR simulation. (a) initial (vertical) position. NTX is bound to Y3.33 and hydrogen bonds linking TM7 with TM3 (D3.32-Y7.43) and TM7 with TM6 (W6.48 - S7.46) are unbroken. (b) final (horizontal) position. NTX is bound to H6.52 and both hydrogen bonds of TM7 are broken.

During the aforementioned changes we observed also a break of a hydrogen bond linking TM6 and TM7: W6.48 S7.46. Such a link exists also in $\beta_{2}$ AR [29] but it is mediated by one water molecule. However, it does not exist in rhodop- sin. Therefore, it seems that this event is not general to GPCRs and plays an auxiliary role during activation of opioid receptors (S7.46 is conserved in all opioid receptors).

Influence of the agonist structure on the process of breaking of the 3-7 lock. The smallest of the analyzed ligands, morphine, broke the D3.32-Y7.43 hydrogen bond in one fast event. In the case of N-methyl-morphine the process of breaking of the 3-7 lock lasted 300 ps (from 400 to 700 ps) (Fig. 5) and then this bond was restored several times in a slow manner. Although the N-moiety of N-methylmorphine was bulkier than morphine and located very close to both interacting residues D3.32 and Y7.43, it did not facilitate the break of the TM3-TM7 connection. Thus, a small difference in the structure of the analyzed agonists resulted in a very different behavior exemplified by a difference in the time needed to break the 3-7 lock. Explanation of such behavior and clarification of trends will be possible after analysis of simulations of higher number of different complexes of $\mu \mathrm{OR}$.

Implications for other GPCRs. The key residues D3.32, Y7.43, Y3.33 and H6.52, participating in formation of the 37 lock and also in the proposed binding modes for $\mu \mathrm{OR}$, exist in all opioid receptors so the binding of antagonists to Y3.33 and agonists to H6.52 may be also possible in $\delta \mathrm{OR}$ and $\kappa O R$. An influence of agonist binding on the break of the 3-7 lock and the subsequent rotamer toggle switch needs to be investigated further for all opioid receptors.

The structures of activated intermediates of rhodopsin, lumirhodopsin, obtained by Nakamichi et al. [64] and of a deprotonated intermediate by Salom et al. [65] shed some light on how the structure of a GPCR is changing upon activation although both the ionic lock (DRY switch) and the rotamer toggle switch remain in resting states, in spite of the fact that retinal is in activated (all-trans) conformation. Interestingly, the 3-7 lock is broken in the lumirhodopsin structure. On the other hand in a recently published structure of $\beta_{2} \mathrm{AR}$ the DRY switch is in the ON state (a connection between TM3 and TM6 is broken) and this is due to binding of an inverse agonist. The hydrogen bond D3.32-Y7.43 exists also in $\beta_{2} \mathrm{AR}$ and is unbroken when an inverse agonist is bound [29]. There is an additional residue on TM7 in $\beta_{2} A R$, N7.39 (not present in opioid receptors), which binds to the protonated amine of a ligand and connects it via interactions with D3.32 to TM3. In unliganded receptor N7.39 probably binds directly to D3.32. So Y7.43 is not critical in $\beta_{2} A R$ and mutations of this residue alone do not lead to constitutive activation of the receptor.

\section{CONCLUSIONS}

Using homology modeling, simulated annealing and molecular dynamics of the $\mu$ opioid receptor complexes we proposed distinct binding modes of opioids carrying the same structural motif - tyramine. Although they bind to the same binding pocket and the protonated amine interacts with D3.32, the antagonist's phenolic $\mathrm{OH}$ group tends to bind Y3.33 whereas agonist's H6.52. All studied agonists broke the 3-7 lock (the hydrogen bond D3.32-Y7.43 in $\mu \mathrm{OR}$ ). Moreover, an antagonist NTX, when restrained to bind H6.52, was also able to break this connection and to induce a rotamer toggle switch. Because of small temporal difference between these events both switches may be interdependent 
or even constitute a larger multicomponent switch. Nanosecond timescale used in conducted simulations is small compared to full activation time of the receptor. However, we investigated only the specific action of switches and the ligands were located in the binding site already so usage of this timescale is justified.

Subsequent simulations and experiments are needed to verify the proposed timeline of observed transitions as well as for checking the interdependence (or independence) of activation switches. Such simulations would involve other ligands and also other opioid receptors. Analysis of opioid receptor mutants, especially involving key residues Y3.33 and H6.52 (important for the proposed binding modes of antagonists and agonists) as well as D3.32 and Y7.43 (constituting the 3-7 lock) is needed to investigate these binding modes and transitions in detail. Combination of simulations and experimental data will also help to explain further activation steps and to find possible new switches occurring during agonist action and probably also during the action of partial and inverse agonists.

\section{ACKNOWLEDGEMENTS}

The work was supported by the Polish Ministry of Science and Higher Education (grant numbers N N301 203833 and N N401 1401 33). M. Kolinski acknowledges the School of Molecular Medicine for a stipend supporting his $\mathrm{PhD}$ study. The modeling was partly done at the ICM Computer Centre, Warsaw University, Poland.

\section{SUPPORTING MATERIAL}

Figures: the sequence alignments, Ramachandran plots, RMSD plots, density distribution plots, a view of the receptor in the membrane in a periodic box, and plots of selected distances and angles in $\mu \mathrm{OR}$ complexes. Movies: four movies showing the most important fragments of molecular dynamics simulations of all analyzed complexes of $\mu \mathrm{OR}$.

\section{Click here for Supporting Material}

\section{Abbreviations}

$\begin{array}{ll}\beta_{2} \mathrm{AR} & =\beta_{2} \text { adrenergic receptor } \\ \beta \text {-FNA } & =\beta \text {-funaltrexamine } \\ \mu \mathrm{OR} & =\mu \text { opioid receptor } \\ \mathrm{GPCR} & =\text { G-protein-coupled receptor } \\ \mathrm{MD} & =\text { Molecular dynamics } \\ \mathrm{NTX} & =\text { Naltrexone } \\ \mathrm{PDB} & =\text { Protein Data Bank } \\ \mathrm{RMSD} & =\text { Root mean square displacement } \\ \mathrm{TM} & =\text { Transmembrane domain }\end{array}$

\section{REFERENCES}

[1] Mirzadegan T, Benko G, Filipek S, Palczewski K. Sequence analyses of G-protein-coupled receptors: similarities to rhodopsin. Biochemistry 2003; 42: 2759-67.

[2] Ballesteros J, Palczewski K. G protein-coupled receptor drug discovery: implications from the crystal structure of rhodopsin. Curr Opin Drug Discov Dev 2001; 4: 561-74.

[3] Bartfai T, Benovic JL, Bockaert J, et al. The state of GPCR research in 2004. Nat Rev Drug Discov 2004; 3: 574-626.
[4] Sautel M, Milligan G. Molecular manipulation of G-proteincoupled receptors: A new avenue into drug discovery. Curr Med Chem 2000; 7: 889-96.

[5] Pierce KL, Premont RT, Lefkowitz RJ. Seven-transmembrane receptors. Nat Rev Mol Cell Biol 2002; 3: 639-50.

[6] Schioth HB, Fredriksson R. The GRAFS classification system of G-protein coupled receptors in comparative perspective. Gen Comp Endocrinol 2005; 142: 94-101.

[7] Waldhoer M, Bartlett SE, Whistler JL. Opioid receptors. Annu Rev Biochem 2004; 73: 953-90.

[8] Corbett AD, Henderson G, McKnight AT, Paterson SJ. 75 years of opioid research: the exciting but vain quest for the Holy Grail. Br J Pharmacol 2006; 147: S153-S62.

[9] Kane BE, Svensson B, Ferguson DM. Molecular recognition of opioid receptor ligands. AAPS J 2006; 8: E126-E37.

[10] Sykes KT, White SR, Hurley RW, et al. Mechanisms responsible for the enhanced antinociceptive effects of mu-opioid receptor agonists in the rostral ventromedial medulla of male rats with persistent inflammatory pain. J Pharmacol Exp Ther 2007; 322: 813-21.

[11] Roy S, Barke RA, Loh HH. MU-opioid receptor-knockout mice: role of mu-opioid receptor in morphine mediated immune functions. Mol Brain Res 1998; 61: 190-4.

[12] Philippe D, Chakass D, Thuru X, et al. Mu opioid receptor expression is increased in inflammatory bowel diseases: implications for homeostatic intestinal inflammation. Gut 2006; 55: 815-23.

[13] Spijker P, Vaidehi N, Freddolino PL, Hilbers PAJ, Goddard WA. Dynamic behavior of fully solvated beta 2 -adrenergic receptor, embedded in the membrane with bound agonist or antagonist. Proc Natl Acad Sci USA 2006; 103: 4882-7.

[14] Bhattacharya S, Hall SE, Li H, Vaidehi N. Ligand-stabilized conformational states of human beta(2) adrenergic receptor: Insight into G-protein-coupled receptor activation. Biophys J 2008; 94: 2027-42.

[15] Li YY, Zhu FQ, Vaidehi N, et al. Prediction of the 3D structure and dynamics of human DP G-protein coupled receptor bound to an agonist and an antagonist. J Am Chem Soc 2007; 129: 10720-31.

[16] Heo JY, Han SK, Vaidehi N, et al. Prediction of the 3D structure of FMRF-amide neuropeptides bound to the mouse MrgC11 GPCR and experimental validation. ChemBioChem 2007; 8: 1527-39.

[17] Peng JYC, Vaidehi N, Hall SE, Goddard WA. The predicted 3D structures of the human M1 muscarinic acetylcholine receptor with agonist or antagonist bound. ChemMedChem 2006; 1: 878-90.

[18] Vaidehi N, Schlyer S, Trabanino RJ, et al. Predictions of CCR1 chemokine receptor structure and BX 471 antagonist binding followed by experimental validation. J Biol Chem 2006; 281: 2761320.

[19] Fanelli F, De Benedetti PG. Inactive and active states and supramolecular organization of GPCRs: insights from computational modeling. J Comput Aided Mol Des 2006; 20: 449-61.

[20] Filipek S, Teller DC, Palczewski K, Stenkamp R. The crystallographic model of rhodopsin and its use in studies of other G protein-coupled receptors. Annu Rev Biophys Biomol Struct 2003; 32: 375-97.

[21] Fanelli F, De Benedetti PG. Computational Modeling approaches to structure-function analysis of $\mathrm{G}$ protein-coupled receptors. Chem Rev 2005; 105: 3297-351.

[22] Kobilka BK, Deupi X. Conformational complexity of G-proteincoupled receptors. Trends Pharmacol Sci 2007; 28: 397-406.

[23] Kobilka BK. G protein coupled receptor structure and activation. Biochim Biophys Acta Biomembr 2007; 1768: 794-807.

[24] Hubbell WL, Altenbach C, Hubbell CM, Khorana HG. Rhodopsin structure, dynamics, and activation: A perspective from crystallography, site-directed spin labeling, sulfhydryl reactivity, and disulfide cross-linking. Adv Protein Chem 2003; 63: 243-90.

[25] Filipek S, Stenkamp RE, Teller DC, Palczewski K. G proteincoupled receptor rhodopsin: A prospectus. Annu Rev Physiol 2003; 65: 851-79.

[26] Palczewski K, Kumasaka T, Hori T, et al. Crystal structure of rhodopsin: A G protein-coupled receptor. Science 2000; 289: 73945.

[27] Okada T, Sugihara M, Bondar AN, et al. The retinal conformation and its environment in rhodopsin in light of a new 2.2 angstrom crystal structure. J Mol Biol 2004; 342: 571-83.

[28] Cherezov V, Rosenbaum DM, Hanson MA, et al. High-resolution crystal structure of an engineered human beta(2)-adrenergic G protein-coupled receptor. Science 2007; 318: 1258-65. 
[29] Rosenbaum DM, Cherezov V, Hanson MA, et al. GPCR engineering yields high-resolution structural insights into beta(2)-adrenergic receptor function. Science 2007; 318: 1266-73.

[30] Rasmussen SGF, Choi HJ, Rosenbaum DM, et al. Crystal structure of the human beta(2) adrenergic G-protein- coupled receptor. Nature 2007; 450: 383-7.

[31] Zhang Y, Sham YY, Rajamani R, Gao JL, Portoghese PS. Homology modeling and molecular dynamics simulations of the $\mathrm{Mu}$ opioid receptor in a membrane-aqueous system. ChemBioChem 2005; 6: 853-9.

[32] Pogozheva ID, Przydzial MJ, Mosberg HI. Homology modeling of opioid receptor-ligand complexes using experimental constraints. AAPS J 2005; 7: E434-E48.

[33] Ballesteros JA, Weinstein H. Integrated methods for the construction of three-dimensional models and computational probing of structure-function relations in $\mathrm{G}$ protein-coupled receptors. Methods Neurosci 1995; 25: 366-428.

[34] Portoghese PS, Sultana M, Takemori AE. Design of peptidomimetic delta opioid receptor antagonists using the messageaddress concept. J Med Chem 1990; 33: 1714-20.

[35] Van der Spoel D, Lindahl E, Hess B, et al. GROMACS: Fast, flexible, and free. J Comput Chem 2005; 26: 1701-18.

[36] Berger O, Edholm O, Jahnig F. Molecular dynamics simulations of a fluid bilayer of dipalmitoylphosphatidylcholine at full hydration, constant pressure, and constant temperature. Biophys J 1997; 72: 2002-13.

[37] van der Spoel D, van Maaren PJ, Berendsen HJC. A systematic study of water models for molecular simulation: Derivation of water models optimized for use with a reaction field. J Chem Phys 1998; 108: 10220-30.

[38] Darden T, York D, Pedersen L. Particle mesh Ewald: An N $\cdot \log (\mathrm{N})$ method for Ewald sums in large systems. J Chem Phys 1993; 98: 10089-92.

[39] Thompson JD, Higgins DG, Gibson TJ. CLUSTAL W: improving the sensitivity of progressive multiple sequence alignment through sequence weighting, position-specific gap penalties and weight matrix choice. Nucleic Acids Res 1994; 22: 4673-80.

[40] Fritze O, Filipek S, Kuksa V, et al. Role of the conserved $\operatorname{NPxxY}(\mathrm{x})_{(5,6)} \mathrm{F}$ motif in the rhodopsin ground state and during activation. Proc Natl Acad Sci USA 2003; 100: 2290-5.

[41] Sali A, Potterton L, Yuan F, vanVlijmen H, Karplus M. Evaluation of comparative protein structure modeling by MODELLER. Proteins $1995 ; 23: 318-26$.

[42] Sanchez R, Sali A. Evaluation of comparative protein structure modeling by MODELLER-3. Proteins 1997: 50-8.

[43] Hess B, Bekker H, Berendsen HJC, Fraaije J. LINCS: A linear constraint solver for molecular simulations. J Comput Chem 1997; 18: 1463-72.

[44] Laskowski RA, MacArthur MW, Moss DS, Thornton JM. PROCHECK: A program to check the stereochemical quality of protein structures. J Appl Cryst 1993; 26: 283-91.

[45] Morris AL, MacArthur MW, Hutchinson EG, Thornton JM. Stereochemical quality of protein structure coordinates. Proteins 1992; 12: 345-64.

[46] Bowie JU, Luthy R, Eisenberg D. A method to identify protein sequences that fold into a known three-dimensional structure. Science 1991; 253: 164-70.

[47] Luthy R, Bowie JU, Eisenberg D. Assessment of protein models with three-dimensional profiles. Nature 1992; 356: 83-5.
[48] Bayly CI, Cieplak P, Cornell WD, Kollman PA. A well-behaved electrostatic potential based method using charge restraints for deriving atom-centered charges: the RESP model. J Phys Chem 1993; 97: 10269-80.

[49] Krieger E, Darden T, Nabuurs SB, Finkelstein A, Vriend G. Mak ing optimal use of empirical energy functions: Force-field parameterization in crystal space. Proteins 2004; 57: 678-83.

[50] Koradi R, Billeter M, Wuthrich K. MOLMOL: A program for display and analysis of macromolecular structures. J Mol Graph 1996; 14: 51-5.

[51] Humphrey W, Dalke A, Schulten K. VMD: Visual molecular dynamics. J Mol Graph 1996; 14: 33-8.

[52] Fowler CB, Pogozheva ID, LeVine H, Mosberg HI. Refinement of a homology model of the mu-opioid receptor using distance constraints from intrinsic and engineered zinc-binding sites. Biochemistry 2004; 43: 8700-10.

[53] Befort K, Tabbara L, Kling D, Maigret B, Kieffer BL. Role of aromatic transmembrane residues of the delta-opioid receptor in ligand recognition. J Biol Chem 1996; 271: 10161-8.

[54] Spivak CE, Beglan CL, Seidleck BK, et al. Naloxone activation of mu-opioid receptors mutated at a histidine residue lining the opioid binding cavity. Mol Pharmacol 1997; 52: 983-92.

[55] Kim JM, Altenbach C, Kono M, et al. Structural origins of constitutive activation in rhodopsin: Role of the K296/E113 salt bridge. Proc Natl Acad Sci USA 2004; 101: 12508-13.

[56] Chelikani P, Hornak V, Eilers M, et al. Role of group-conserved residues in the helical core of beta(2)-adrenergic receptor. Proc Natl Acad Sci USA 2007; 104: 7027-32.

[57] Elling CE, Frimurer TM, Gerlach LO, et al. Metal ion site engineering indicates a global toggle switch model for seventransmembrane receptor activation. J Biol Chem 2006; 281: 17337 46.

[58] Lei S, Liapakis G, Xu R, et al. beta(2) adrenergic receptor activation - Modulation of the proline kink in transmembrane 6 by a rotamer toggle switch. J Biol Chem 2002; 277: 40989-96.

[59] Befort K, Zilliox C, Filliol D, Yue SY, Kieffer BL. Constitutive activation of the delta opioid receptor by mutations in transmembrane domains III and VII. J Biol Chem 1999; 274: 18574-81.

[60] Vilardaga JP. Switching modes for G protein-coupled receptor activation. Nat Chem Biol 2006; 2: 395-6.

[61] Yao XJ, Parnot C, Deupi X, et al. Coupling ligand structure to specific conformational switches in the beta(2)-adrenoceptor. Nat Chem Biol 2006; 2: 417-22.

[62] Azzi M, Charest PG, Angers S, et al. beta-Arrestin-mediated activation of MAPK by inverse agonists reveals distinct active conformations for $\mathrm{G}$ protein-coupled receptors. Proc Natl Acad Sci USA 2003; 100: 11406-11.

[63] Audet N, Paquin-Gobeil M, Landry-Paquet O, Schiller PW, Pineyro G. Internalization and Src activity regulate the time course of ERK activation by delta opioid receptor ligands. J Biol Chem 2005; 280: 7808-16.

[64] Nakamichi H, Okada T. Local peptide movement in the photoreaction intermediate of rhodopsin. Proc Natl Acad Sci USA 2006; 103 12729-34.

[65] Salom D, Lodowski DT, Stenkamp RE, et al. Crystal structure of a photoactivated deprotonated intermediate of rhodopsin. Proc Natl Acad Sci USA 2006; 103: 16123-8. 